\title{
Adaptation or constraint? Reference-dependent scatter in honey bee dances
}

\author{
David A. Tanner • P. Kirk Visscher
}

Received: 6 October 2009 /Revised: 26 January 2010 / Accepted: 3 February 2010 /Published online: 27 February 2010

(C) The Author(s) 2010. This article is published with open access at Springerlink.com

\begin{abstract}
The waggle dance of the honey bee is used to recruit nest mates to a resource. Dancer bees, however, may indicate many directions within a single dance bout; we show that this scatter in honey bee dances is strongly dependent on the sensory modality used to determine a reference angle in the dance. Dances with a visual reference are more precise than those with a gravity reference. This finding undermines the idea that scatter is introduced into dances, which the bees could perform more precisely, in order to spread recruits out over resource patches. It also calls into question reported interspecific differences that had been interpreted as adaptations of the dance to different habitats. Our results support a non-adaptive hypothesis: that dance scatter results from sensory and performance constraints, rather than modulation of the scatter by the dancing bee. However, an alternative adaptive hypothesis cannot be ruled out.
\end{abstract}

Keywords Apis mellifera - Divergence angle · Constraint . Adaptation · Communication

Communicated by: M. Giurfa

D. A. Tanner $(\bowtie)$

Biology Department, Utah State University,

5305 Old Main Hill,

Logan, UT 84322, USA

e-mail: dtanner@biology.usu.edu

P. K. Visscher

Entomology Department, University of California, Riverside,

Riverside, CA 92521, USA

e-mail: visscher@ucr.edu

\section{Introduction}

Ever since Darwin or perhaps Aesop, observed patterns in the natural world have invited conjecture as to how they benefit the organism displaying them. Not all traits are necessarily adaptive, however, and some patterns may arise in adaptive or non-adaptive ways (Gould and Lewontin 1979; Gould and Vrba 1982). Even traits initially present for non-adaptive reasons may be further tuned by natural selection, becoming exaptations (Gould and Vrba 1982).

Honey bee dances

The dance language of the honey bees (Apis spp.) is one of the best known communicative adaptations among animals and has been extensively studied (von Frisch 1967; Gould 1976; Seeley 1995), though only recently tested as an adaptation itself (Sherman and Visscher 2002; Dornhaus and Chittka 2004). Honey bee dances encode the direction and distance to the resource they advertise (von Frisch 1967); these resources include food, water, pollen, and potential nesting sites. A bee learns the angle of flight from her nest to the resource, relative to the current sun azimuth (and updates it for sun movement thereafter, von Frisch 1967). When Apis mellifera dances, she encodes the flight angle as the dance angle of orientation and movement during the waggle-run portion of the dance (see Fig. 1), relative to a directional reference, which may vary. Bees dancing on vertical combs inside a dark cavity use the direction upwards as a reference and detect it by the gravitational deflection of their head and gaster against mechanoreceptor hairs located in the joints between these and the thorax (Lindauer 1971). When the sun is visible to a dancer, she uses the projection of the sun direction on the surface on which she is dancing as a reference. If she sees 


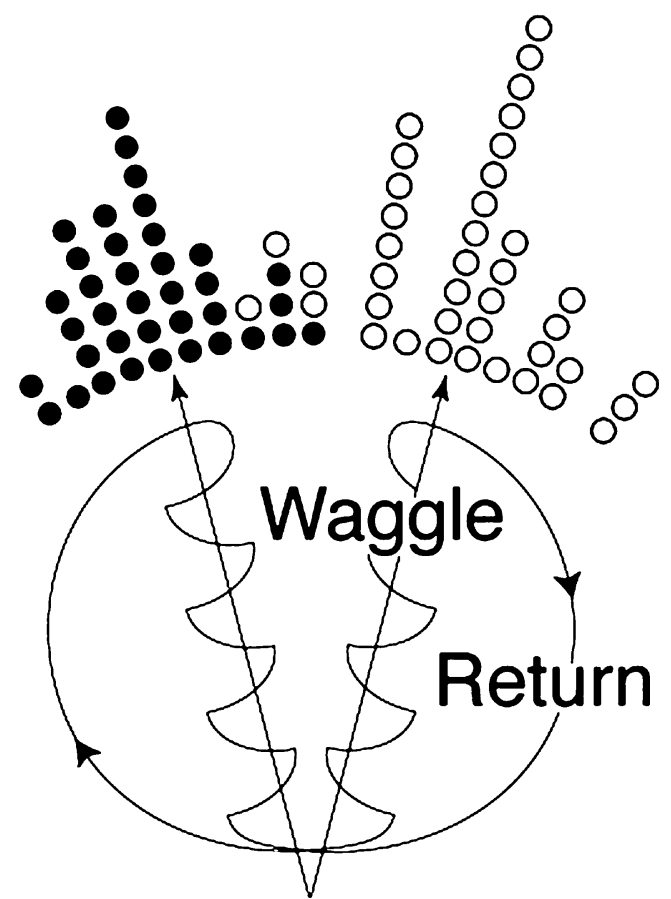

Fig. 1 Dances of five honey bees for a nectar resource located $200 \mathrm{~m}$ from the hive. Circles represent the direction a bee moved during each waggle run following a left turn (circle) and right turn (filled circle), respectively. Arrows indicate the means of these two distributions. The angular difference between successive waggle runs (left-right or right-left) is the divergence angle

UV polarization patterns in the blue sky, she infers the sun direction from these and uses that as a reference or uses an intermediate between the inferred sun direction and upward (von Frisch 1967).

\section{Divergence angle}

The dance angle within a dance, however, is not consistent, especially for dances to nearby resources. Before beginning a waggle run, the bee does not turn as far as needed to reach the expected dance angle, so that alternate waggle runs indicate angles on either side of the proper flight direction, with the angle between them termed the "divergence angle" (Towne 1985; Towne and Gould 1988; Weidenmuller and Seeley 1999; Gardner et a. 2007). The resulting pattern is not normally distributed scatter about the "ideal" direction, but is bimodal (Fig. 1), composed of two somewhatoverlapping distributions. Gardner et al. (2007) analyzed these distributions; they termed the scatter within the waggle runs following either left or right turns (i.e., the right or left hump of the distribution) the "variance." In this paper, to avoid confusing that term with the more familiar statistical variance, which might apply to the whole distribution, we term it "within-side variance." The divergence angle (the separation of the humps of the bimodal distribution) decreases with distance indicated in the dance (longer flight distances result in longer waggle runs, and also smaller divergence angles, Towne and Gould 1988). This aspect of honey bee dances presents an interesting evolutionary problem: if the function of the dance is to direct nestmates to the location of food and other resources, why has inaccuracy in the indication of the direction of those resources persisted? There are two classes of explanations of this: mechanistic constraints and adaptive function.

\section{Constraint}

At short distances, bees perform a round dance with less obvious waggle runs, though Kirchner et al. (1988) demonstrated that bees performing round dances do produce a sound signal and waggling motion at the point in the dance when they are oriented in the direction that would indicate the resource direction, just as they do during the waggle run of the waggle dance performed for more distant resources. At intermediate resource distances, very short waggle runs are performed and the dance pattern becomes sickle shaped and finally approximates the shape of an upper case phi or theta, with waggle angles after the left and right turn coinciding. In Fig. 1 the sickle of the divergence angle is nearing closure; beyond about $400 \mathrm{~m}$ the divergence angle is less than the right or left within-side variance (von Frisch 1967, pp. 61; Towne 1985). Karl von Frisch, who first decoded the waggle dance, viewed the gradually closing divergence as a transition, and pointed out (von Frisch 1967) that "the line, which halves the divergence angle, points the direction to the feeding place approximately just as precisely as with the uniform, straight-line waggle-run."

Edrich (1975) explained the divergence angle as a result of a dancing bee turning until the match between her learned flight angle and the gravity angle was within a certain threshold, and then starting her waggle run. Turning right she would begin at a more-counterclockwise angle (a numerically lower angle in the increasing-clockwise convention of representing angles), and turning left she would begin at more-clockwise angle (numerically greater). He reported that as a vertical comb surface was tilted toward the horizontal, the divergence angle increased, and explained this, and the decreasing divergence for more distant sites, as resulting from the perceived gravity angle and the learned flight angle both having imprecision. In his paper, Edrich (ibid.) refers to the "width of angles" as an index of precision, i.e., the wider the angle, the greater the imprecision. He suggests that there is imprecision in both dance angle, and the flight angle (perhaps due to inherent imprecision in the way it is calculated). Lower comb angles (i.e., a more horizontal comb) would increase the width (decrease the precision) of the gravity angle distribution, and longer flights would decrease the width (increase the precision) of the flight angle distribution. Bees measure 
their angle relative to gravity from the deflection of multiple sensory hairs located in the articulation between head and thorax and thorax and abdomen (Lindauer 1971). Since the bee is constantly moving, it is perhaps not surprising that measuring of gravity angle would have a substantial variance. Thus, Edrich (ibid.) interpreted the divergence angle as a result of constraint on the bees' precision in measuring angles.

\section{Function}

The scatter in honey bee dances has been explained as an adaptation to spread recruits over patches of flowers (Wilson 1962; Gould 1975; Towne 1985; Towne and Gould 1988), and several details of the dance within and between species have been cited as support for this "tuned-error hypothesis." The tuned-error hypothesis interprets the divergence angle as being modulated by the dancing bees to indicate a more-or-less constant patch size at different distances (regardless of whether the resource advertised was collected in a patch or at a point source such as syrup feeder). Apis cerana, an Asian temperate and tropical bee, and the Asian tropical species Apis florea and Apis dorsata have been reported to have smaller divergence angles than A. mellifera (whose range includes temperate and tropical habitats in Europe and Africa). This difference has been cited as additional evidence for the tuned-error hypothesis, invoking an additional hypothesis that tropical bees more often forage on highly clumped food sources where spreading recruits over a patch would be less advantageous (Towne 1985; Towne and Gould 1988). There is little data on whether these resource-dispersion differences are in fact typical of the native habitats of the various Apis (but see Dornhaus and Chittka 2004). Certainly not all resources used by temperate bees occur in large patches, and home sites in particular, which are also advertised by dances, are always point sources: i.e., a single knothole in a hollow tree. Towne (1985) measured the divergence angle of bees on a swarm dancing to advertise nest sites, and found it no different than in dances in a hive for nectar. Weidenmuller and Seeley (1999) also compared these and reported that dances for nest sites were more precise than those for nectar. However, their results may have confounded effects of dance substrate with those of the resource advertised (Tanner and Visscher 2006). Tanner and Visscher (2006) reported that dance substrate affected the divergence angle, but that the context (swarm versus observation hive, or nectar versus nest sites on a swarm) did not.

\section{Constraint versus function}

The tuned-error hypothesis concerns the adaptive function of the divergence angle. Constraints concern the mecha- nism of performance of the dance. These two explanations are at different levels of analysis and thus cannot be mutually exclusive alternatives to each other. The divergence angle could arise from mechanistic constraints and be functionally useful (and thus limit selection for overcoming constraints to increased precision). However, if constraints explain the origin of all the features of the divergence angle phenomenon, it would no longer be an adaptation, since it was not shaped by natural selection. If, however, bees were shown capable of performing their dances more precisely, but did not, or if they performed them more precisely when doing one thing (say househunting) than another, and these differences were not due to differences in the constraints, it would strengthen the case for the observed patterns being an adaptation.

On the other hand, if bees are dancing as well as they can, and scatter is introduced by constraints on their ability to dance precisely, the resulting error could still function adaptively to spread out recruits (if that were advantageous, which we find questionable), but finding that a mechanistic constraint underlies the observed patterns would weaken the idea that the scatter is tuned by natural selection as an adaptation.

We performed this study to test the hypothesis that bees were introducing error into the dance beyond that imposed by sensory constraints against an alternative hypothesis that if they could dance more precisely because of reduction of the constraints, they would do so. We tested this by comparing dance precision when the bees are provided a different sensory reference for their dances.

\section{Methods}

To determine whether the reference that bees use to orient their dances affects dance precision, we trained bees to feeders containing $2 \mathrm{M}$ sucrose located 200, 300, and $400 \mathrm{~m}$ from the hive, and compared mean divergence angle in dances by individually marked bees returning from these feeders. We did this as they danced on a vertical comb in the dark, on a vertical comb with a view of the blue sky, or on a horizontal comb with a view of the blue sky.

\section{Bees and observation hive}

Each colony in this study consisted of approximately 2,500 honey bees of mixed European lineage. We housed each colony in an observation hive that could be rotated from a vertical to horizontal orientation without disturbing them (Fig. 2). We placed the observation hives in a $1.5 \times 1.5 \times 2 \mathrm{M}$ modular wood hut from which the roof and walls could be removed to allow the bees a view of the blue sky (Fig. 3). The bees gained access to the hive 


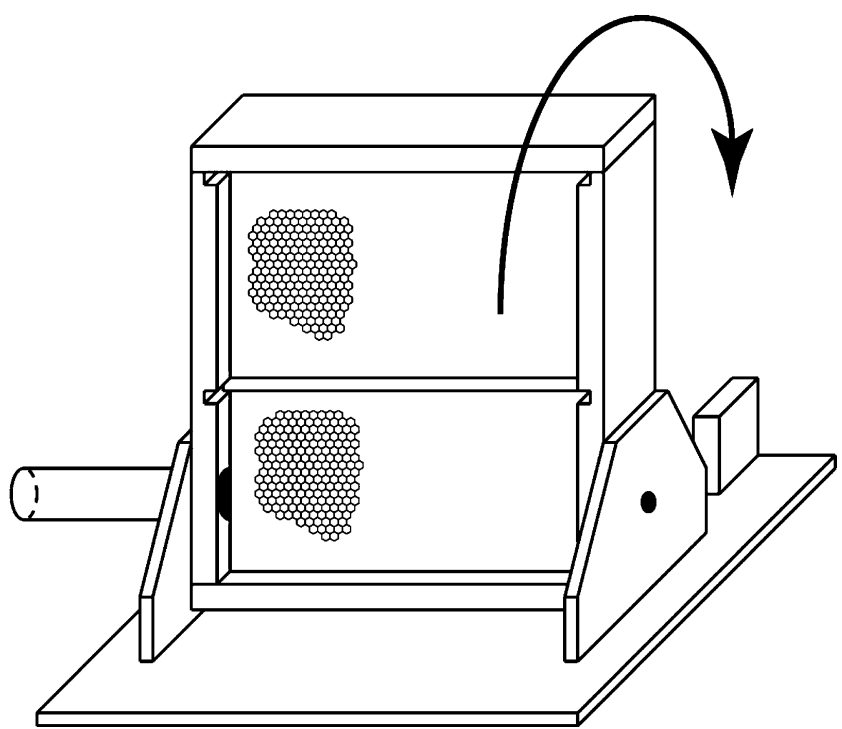

Fig. 2 Observation hive used in this experiment. The surface of the hive was covered with plate glass (colonies 1 and 2) or wire mesh (colony 3 ) to facilitate the video recording of bee dances. During the horizontal treatment, the hive was rotated, using the entrance tube as one pivot point and a screw extending into the hive body as the second

through short length of PVC tube that extended from an entrance hole to the hive and through the wall of the wood hut. We fitted the entrance hole with a wooden wedge that directed all bees returning to the hive to the side of the comb facing a digital video camera (Sony DCR-TR50 DV).

Training and data collection

We trained bees to collect nectar from feeders located 200, 300 , and $400 \mathrm{~m}$ from the hive by placing a feeding jar, containing a $2 \mathrm{M}$ sucrose solution scented with $15 \mu \mathrm{L} / \mathrm{L}$ anise oil, next to the entrance tube to the hive. Once bees were actively feeding on the solution, we gradually moved the feeder $200 \mathrm{~m}$ from the hive. Each bee that visited the feeder was individually marked with a colored and numbered plastic tag, and the dances of these bees were video-recorded at the hive. Once a marked bee performed a dance consisting of at least 20 consecutive waggle runs, she was captured at the feeder in plastic bag and placed in a cooler. Bees were captured and placed in a cooler to decrease the amount of competition at the feeder, which may affect the probability of bees dancing (personal observation). After the dances of at least 13 individual bees from each colony were recorded, we released the bees from the cooler and removed three walls and the roof from the hut, exposing the vertical dance floor of the hive to the blue sky. After recording dances performed on the vertical dance floor while dancer bees viewed the blue sky, we again released the bees that were captured after successfully dancing and rotated the hive horizontal. Bees that were collected and released rarely danced for succeeding treatments. After collecting sufficient data from these treatments, we moved the feeder 300 and then $400 \mathrm{~m}$ from the hive, following the same protocol at each distance.

We conducted this experiment with three colonies of bees. Colonies 1 and 2 had $3 \mathrm{~mm}$-thick plate glass sides; UV polarization patterns penetrated this glass sufficiently that dances were well oriented in horizontal hives, where they would be disoriented without the skylight patterns (von Frisch 1967; Sherman and Visscher 2002), but the glass undoubtedly absorbed much UV light, especially in the oblique view of the sky in the vertical hive, so in colony 3 it was replaced by wire mesh, and in this colony we recorded dances on vertical combs in the dark and with a view of the sky.

\section{Data analysis}

We analyzed the dances of at least 13 individual bees for each treatment at each distance for each colony, using only dances in which a bee performed at least 14 consecutive waggle runs. In frame by-frame video playback, we measured the angle of each waggle run and calculated the

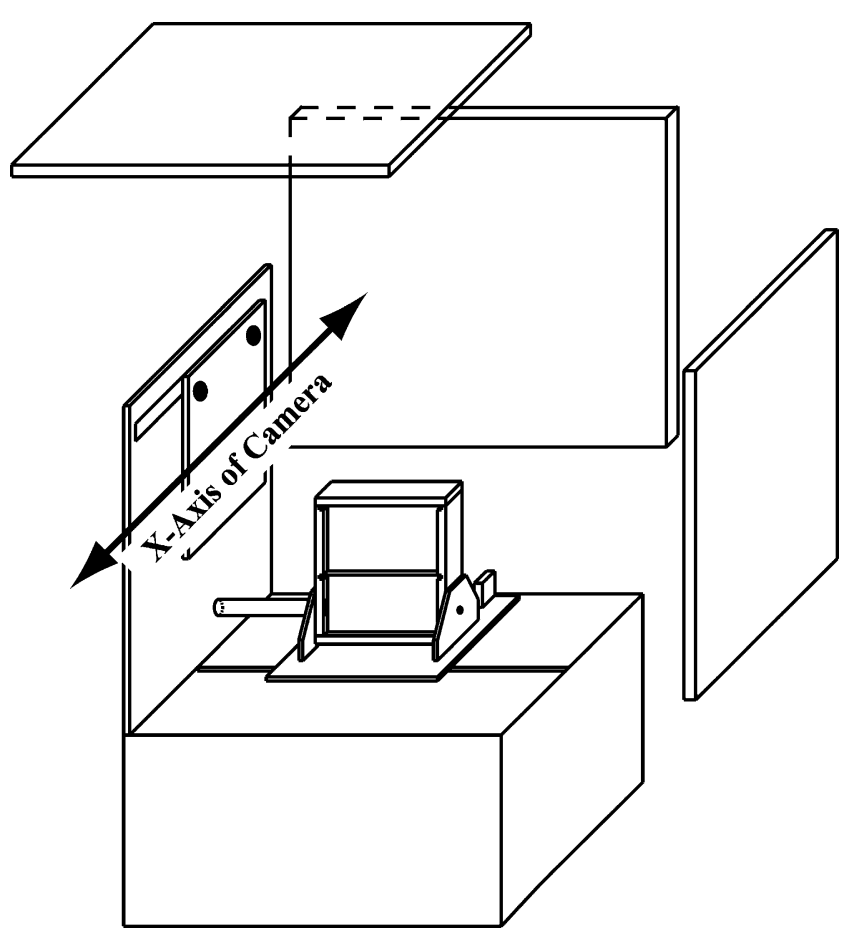

Fig. 3 Hut that housed the observation hive. During the "light" treatments, three walls and the roof were removed from the hut. During the "horizontal" treatment, a camera and tripod were mounted on a plywood sled on the remaining wall. The sled moved along the $X$-axis of the wall, which allowed us to videotape bees dancing on different areas of the hive without introducing parallax into the video 
absolute value of the angular difference between each run and the next. For each bee, we averaged these differences, and then performed ANOVAs testing for treatment and distance effects. We performed post-hoc Tukey-Kramer tests where significant effects or interactions were detected. Weidenmuller and Seeley (1999) used a different measure of mean divergence angle, taking the mean of all dance angles following a right turn or a left turn, and then calculating a difference of these means, instead of the mean of the differences we used. We use the mean of differences approach since it is more directly a measure of what a bee experiences when following a limited number of circuits of the dance. However, both approaches yield very similar numbers for the mean divergence angle.

\section{Results}

Our analysis shows a significant effect of orientation reference on the magnitude of divergence angle in the

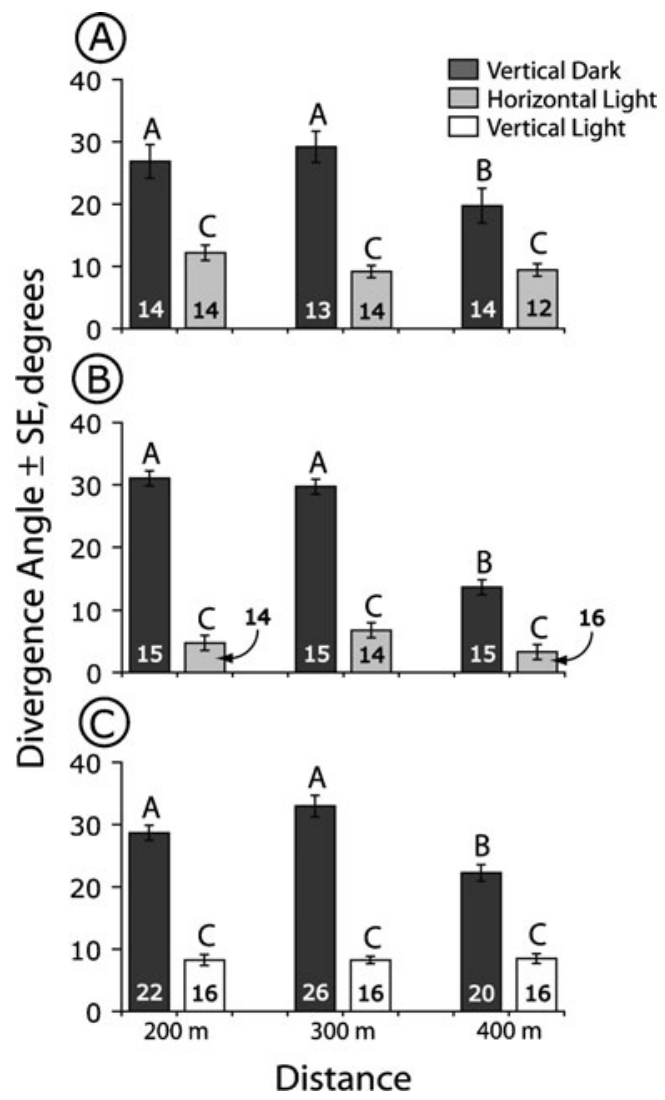

Fig. 4 Mean divergence angle of honey bee dances using different directional references. a Data from colony 1. b Data from colony 2. c Data from colony 3. Bars within colonies that do not share an uppercase letter are significantly different from one another (TukeyKramer's post-hoc, $P<0.05)$. The numbers inside the bins represent the number of individual bees recorded dances of $A$. mellifera (Fig. 4). In colonies 1, 2, and 3, dances performed with only a gravity reference (i.e., the vertical+dark treatment) have significantly larger divergence angles than do dances performed with a visual reference (i.e., horizontal+skylight or vertical+skylight; [Colony $1 F_{0.05,2}=4.433, P=0.0139$; Colony $2 F_{0.05,2}=$ 9.681, $P<0.001$; and Colony $3 F_{0.05,2}=203.177, P<$ $0.001])$. Distance and distance $\times$ treatment interaction each had a significant effect $(P<0.001)$. The Tukey-Kramer analysis clarifies this effect by showing a significant decrease in divergence angle with distance in the vertical + dark treatment, as noted by many authors (Wilson 1962; von Frisch 1967; Edrich 1975; Gould 1975; Towne 1985; Towne and Gould 1988; Weidenmuller and Seeley 1999), but no such pattern in the horizontal+skylight or vertical +skylight treatments (Fig. 4).

\section{Discussion}

Our results, that bees perform dances more precisely when they have an optical directional reference than when they have only a gravitational reference, suggest that the reference used by the bees for orientation while dancing may constrain the overall accuracy of the dance. This finding supports the hypothesis that $A$. mellifera dance as precisely as they are able and that divergence angle in the dance language results from a sensory constraint. Further, the divergence angle does not change significantly with distance when bees dance with a view of celestial light patterns (Fig. 4). The tuned-error hypothesis was suggested after observing the inverse relationship between divergence angle and the distance of a resource from the hive, which is yet cited as supporting evidence for bees "tuning" dance error (Beekman et al. 2008); since that relationship disappears when the reference for orientation changes, we suggest that it may be an artifact: that bees doing longer dances in the dark improve their orientation as they have more opportunity to measure the direction of gravity (which they must do from deflection of joints that are themselves moving during the dance).

The Asian honey bees reportedly have smaller divergence angle in their dances for a given distance than do A. mellifera (Towne 1985; Towne and Gould 1988). A. florea, however, dances on horizontal surfaces, and $A$. dorsata on vertical ones, both in the open, with a view of the sky. We show that under these conditions $A$. mellifera performs dances with low divergence angle, as well. $A$. cerana nests inside of normally dark cavities, but the small amount of data on the divergence angle in $A$. cerana dances were gathered in unspecified conditions, that may have included a view of the sky from the observation hive, which we found could reduce diver- 
gence angle in A. mellifera (Fig 4c). Thus, while these patterns deserve careful re-examination, the interspecific differences in divergence angle, which were invoked to support that the patterns of divergence angle were likely an adaptation, may also be an artifact of orientation reference differences.

Beekman et al. (2008) report that there is little difference in the spatial scatter in dances for nectar and nest sites by $A$. florea and that this similarity suggests that the honey bees' dance evolved as an adaptation for nest site recruitment first and subsequently for food resources. Further, they suggest that cavity nesting bees' ability to "tune" the imprecision in their dances evolved once the dance was used for nectar, an ability that they claim is evidenced by decreased directional variation over distance. Tanner and Visscher (2006), however, show that there is also no difference in the spatial variation in dances performed for nectar and nest sites by $A$. mellifera, when physiological constraints to dance precision are inhibited, and we here show that the relationship between dance imprecision and distance may be nothing more than an artifact of the sensory signal used for dance orientation (Fig. 4). In light of these data, we strongly suggest that hypotheses regarding the utility of imprecision in the dance, and the dance as an adaptation for nest site recruitment be reassessed.

In conclusion, it seems that the tuned-error hypothesis can find little support in the patterns of changing divergence angle with distance, which first suggested it, nor in tuning the dances differently for different resources, nor in interspecific differences that could correlate with different ecologies in different species of Apis. In light of this evidence, we suggest that adaptation need not be invoked to explain imprecision in the dance language, but that it may be more parsimoniously explained as a sensory constraint.

Acknowledgments We would like to thank Alexis Park and Richard Vetter for their assisting with data collection and Rick Redak and John Klotz for a thoughtful review of this manuscript.

Open Access This article is distributed under the terms of the Creative Commons Attribution Noncommercial License which per- mits any noncommercial use, distribution, and reproduction in any medium, provided the original author(s) and source are credited.

\section{References}

Beekman M, Gloag RS, Even N, Wattanachaiyingchareon W, Oldroyd BP (2008) Dance precision in Apis florea - clues to the evolution of the honeybee dance language? Behav Ecol and Sociobiol 62:1259-1265

Dornhaus A, Chittka L (2004) Why do honey bees dance? Behav Ecol and Sociobiol 55:395-401

Edrich W (1975) The waggle dance of the honey bee: a new formulation. Fortschr der Zoöl 23:20-30

Gardner KE, Seeley TD, Calderone NW (2007) Hypotheses on the adaptiveness or non-adaptiveness of the directional imprecision in the honey bee waggle dance (Hymenoptera: Apidae: Apis mellifera). Entomologia Generalis 29(2/4):285-298

Gould JL (1975) Honey bee recruitment: the dance language controversy. Science (Washington DC) 189:685-693

Gould JL (1976) The dance-language controversy. Q Rev Biol 51:211-244

Gould SJ, Lewontin RC (1979) The spandrels of San Marco and the Panglossian paradigm: a critique of the adaptationist programme. Proc R Soc Lond Ser B 205(1161):581-598

Gould SJ, Vrba E (1982) Exaptation: a missing term in the science of form. Paleobiol 8(1):4-15

Kirchner WH, Lindauer M, Michelsen A (1988) Honeybee dance communication-acoustical indication of direction in round dances. Naturwissenschaften 75:629-630

Lindauer M (1971) Communication among social bees. Harvard University Press, Cambridge

Seeley TD (1995) The wisdom of the hive: the social physiology of honey bee colonies. Harvard University Press, Cambridge

Sherman G, Visscher PK (2002) Honeybee colonies achieve fitness through dancing. Nature (London) 419:920-922

Tanner DA, Visscher PK (2006) Do honey bees tune error in their dancing in nectar foraging and house-hunting? Behav Ecol Sociobiol 59:571-576

Towne WF (1985) The spatial precision and mechanisms of dance communication of honey bees: experimental and comparative studies. Dissertation, Princeton Unversity, Princeton

Towne WF, Gould JL (1988) The spatial precision of the dance communication of honey bees. J Insect Behav 1:129-155

von Frisch K (1967) The dance language and orientation of bees. Harvard University Press, Cambridge

Weidenmuller A, Seeley TD (1999) Imprecision in waggle dances of the honeybee (Apis mellifera) for nearby food sources: error or adaptation? Behav Ecol Sociobiol 46:190-199

Wilson EO (1962) Chemical communication among workers of the fire ant Solenopsis saevissima (Fr. Smith). 2: An information analysis of the odour trail. Anim Behav 10:148-158 